\title{
BMJ Diagnosing Mild Cognitive Impairment Open (MCI) in clinical trials: a systematic review
}

\author{
Blossom Christa Maree Stephan, ${ }^{1}$ Thais Minett, ${ }^{2}$ Emma Pagett, ${ }^{2}$ Mario Siervo, ${ }^{3}$ \\ Carol Brayne, ${ }^{2}$ lan G McKeith ${ }^{3}$
}

To cite: Christa Maree Stephan B, Minett T, Pagett $\mathrm{E}$, et al. Diagnosing Mild Cognitive Impairment (MCI) in clinical trials: a systematic review. BMJ Open 2013;3:e001909.

doi:10.1136/bmjopen-2012001909

- Prepublication history and additional material for this paper are available online. To view these files please visit the journal online (http://dx.doi.org/10.1136/ bmjopen-2012-001909).

Received 15 August 2012 Revised 7 January 2013 Accepted 9 January 2013

This final article is available for use under the terms of the Creative Commons Attribution Non-Commercial 2.0 Licence; see http://bmjopen.bmj.com

${ }^{1}$ Institute of Health and Society, Newcastle University, Newcastle upon Tyne, UK ${ }^{2}$ Department of Public Health and Primary Care, Cambridge University, Cambridge, UK

${ }^{3}$ Institute for Ageing and Health, Newcastle University, Newcastle upon Tyne, UK

Correspondence to Dr Blossom Christa Maree Stephan;

blossom.stephan@ncl.ac.uk

\section{ABSTRACT}

Objective: To describe how criteria for amnestic Mild Cognitive Impairment (aMCl) have been operationalised in randomised controlled clinical trials (RCTs).

Design: Systematic review.

Information sources: EMBASE, PubMed and PSYCHInfo were searched from their inception to February 2012. Electronic clinical trial registries were also searched (February 2012).

Study selection: RCTs were included where participant selection was made using Petersen et al-defined aMCl. There was no restriction on intervention type or the outcome tested.

Data extraction: For each trial, we extracted information on study design, demographics, exclusion criteria and the operationalisation strategy for the five aMCl diagnostic criteria including: (1) memory complaint, (2) normal general cognitive function, (3) memory impairment, (4) no functional impairment and (5) no dementia.

Results: 223 articles and 278 registered trials were reviewed, of which 22 met inclusion criteria. Various methods were applied for operationalising aMCl criteria resulting in variability in participant selection. Memory complaint and assessment of general cognitive function were the most consistently measured criteria. There was large heterogeneity in the neuropsychological methods used to determine memory impairment. It was not possible to assess the impact of these differences on case selection accuracy for dementia prediction. Further limitations include selective and unclear reporting of how each of the criteria was measured.

Conclusions: The results highlight the urgent need for a standardised approach to map aMCl. Lack of uniformity in clinical diagnosis, however, is not exclusively a problem for $\mathrm{MCl}$ but also for other clinical states such as dementia including Alzheimer's disease, Lewy Body, frontotemporal or vascular dementia. Defining a uniform approach to $\mathrm{MCl}$ classification, or indeed for any classification concept within the field of dementia, should be a priority if further trials are to be undertaken in the older aged population based on these concepts.

\section{ARTICLE SUMMARY}

Article focus

- Accurate identification of individuals at risk of dementia or with predementia is important for clinical trial enrolment.

- Diagnosis of predementia is usually made using the amnestic form of Mild Cognitive Impairment (aMCI). While specific criteria for implementation exist, there is no operationalisation protocol.

- Research Question: How have criteria for aMCl been operationalised in randomised controlled clinical trials?

Key messages

- Various methods have been applied for operationalising aMCI criteria in randomised controlled clinical trials resulting in variability in participant selection.

- The results highlight the urgent need for a standardised approach to map aMCI.

- Lack of specific methods for clinical diagnosis is not a problem unique to the field of $\mathrm{MCl}$. Across studies there continues to be inconsistency in the instruments and methodology used to diagnose Alzheimer's disease and Vascular Dementia, including its prodromal stage, Vascular Cognitive Impairment no Dementia. Revision of diagnostic criteria should be a research priority.

Strengths and limitations of this study

- The review focuses on predementia defined using aMCl. However, not all clinical trials on predementia cognitive states have used this definition of $\mathrm{MCl}$.

- We chose to focus on aMCl as this is one of the commonly applied definitions in clinical and research practice.

\section{INTRODUCTION}

As new preventative strategies for dementia are developed, methods to select persons accurately for clinical trial involvement will be needed. In this perspective, Mild Cognitive Impairment (MCI), an intermediate state between normal ageing and dementia, has 
become a focus for trials to prevent or delay progression to Alzheimer's disease. The expectation is that positive results are more likely to be achieved with earlier treatment initiation. ${ }^{12}$ While several different definitions exist for MCI, amnestic Mild Cognitive Impairment (aMCI) as defined by Petersen $e t a \hat{l}^{4}$ is often used in clinical and research practice. However, despite being commonly applied, no standardised method for the operationalisation of each of the five component criteria (figure 1) necessary for an aMCI diagnosis exists, resulting in heterogeneity in diagnostic methods and case ascertainment across studies. Indeed, there are numerous possibilities for the measurement of the five criteria as highlighted in figure 1. The lack of an established diagnostic methodology for identifying cases for clinical trial enrolment is problematic as study-specific participant selection raises questions regarding the nature of the sample selected, while also making cross study comparison and generalisability of findings difficult.

We undertook a systematic review to explore the methods used to classify aMCI cases, defined using Petersen $e t a \hat{l}$ criteria, in randomised controlled clinical trials (RCTs). The focus was on inclusion criteria and variation in the operationalisation of each of the five aMCI component criteria as outlined in figure 1 .

\section{METHODS}

This review has been undertaken with adherence to the PRISMA statement. ${ }^{5}$ The review protocol is available on request.

\section{Search strategy}

EMBASE (including MEDLINE) and PSYCHInfo were searched using the following keywords and using Medical Subject Heading terms: ('mild cognitive impairment' OR MCI) AND ('randomised controlled trial' OR 'randomised controlled trial' OR RCT). Articles were searched from inception to 6 June 2011, with the search updated on 21 February 2012. Web-based searches, using the term 'mild cognitive impairment', were also undertaken in the ISRCTR trial registry (http://www. controlled-trials.com) and on www.clinicaltrials.gov (17 February 2012). Only studies that were published in English were included. Two investigators (BS and TM) independently searched publications using the following inclusion criteria: (1) the study was a RCT; (2) the trial had been completed (was not ongoing or terminated) and results published; (3) the authors report selecting participants using the definition of aMCI as reported in Petersen $e t ~ a l,{ }^{3}$ and could include single or multidomain aMCI subtypes (amendments to criteria were allowed as long as they were stated and Petersen $e t a l^{3}$ was referenced) and (4) the MCI group was analysed separately to the dementia or control groups. The protocol paper or the first publication reporting the primary outcome was selected in case of multiple publications using the same study sample. Titles and abstracts were searched first, followed by the full text of any identified articles. Reviews were also retained and the reference lists of these and each included paper were interrogated. Disagreements were resolved by consensus. Data quality was not assessed as all included studies were RCTs.

\section{Data extraction}

Data on the lead author, date of publication, study design (country, site, sampling framework, duration and intervention), demographics (age and gender distributions), trial exclusion criteria, dementia progression rates, outcomes tested and the methods used to operationalise each of the five component criteria for the diagnosis of aMCI were abstracted by two investigators (EP and TM) and checked by a third (MS).

\section{RESULTS}

A total of 223 articles were identified from the literature search. From the electronic search, 11 trials were identified from the ISRCTR trial registry and 267 from http://www.clinicaltrials.gov. Based on the title-abstract
Figure 1 Petersen criteria for amnestic Mild Cognitive Impairment.
1. Subjective memory complaint (preferably corroborated by an informant) Operationalisation Issues Participant, informant, single question, questionnaire

2. Normal general cognitive function Operationalisation Issues Test selection, use of a cut-off score, adjustments for age, education or prior ability

3. Objective memory impairment Operationalisation Issues Test selection, use of a cut-off score, adjustments for age, education or prior ability

4. Preserved activities of daily living (ADL)

Operationalisation Issues Type of impairment such as instrumental or basic activities of living, degree of difficulty (if any) allowed

5. No dementia 
search, 84 articles were identified for full text review. In total, 22 articles met inclusion criteria and were retained for this review. Figure 2 shows the selection process using the PRISMA (2009) Flow Diagram. As shown in figure 2, articles were mainly excluded as the sample did not appear to be defined using the Petersen $e t a l^{3}$ criteria or had inadequate details to support the use of Petersen $e t a \hat{l}^{3}$ criteria (eg, only stated an objective cognitive deficit), or the article was a review. Online supplementary table S1A summarises the general characteristics, demographics and outcomes tested in each included article. Online supplementary table S1B summarises the operationalisation protocol used for identifying aMCI cases in each trial.

Trial exclusion criteria varied, but were mainly related to cerebrovascular and cardiovascular disease or health and psychiatric-related conditions that could be associated with cognitive decline. There were also differences in the population sampled (clinic vs community), site (single vs multicentre), duration (eg, 90 days to 4 years) and sample demographics (eg, age range: 50-90 years). Interventions included pharmacological agents and supplementation ${ }^{6-17}$ (including: donepezil,

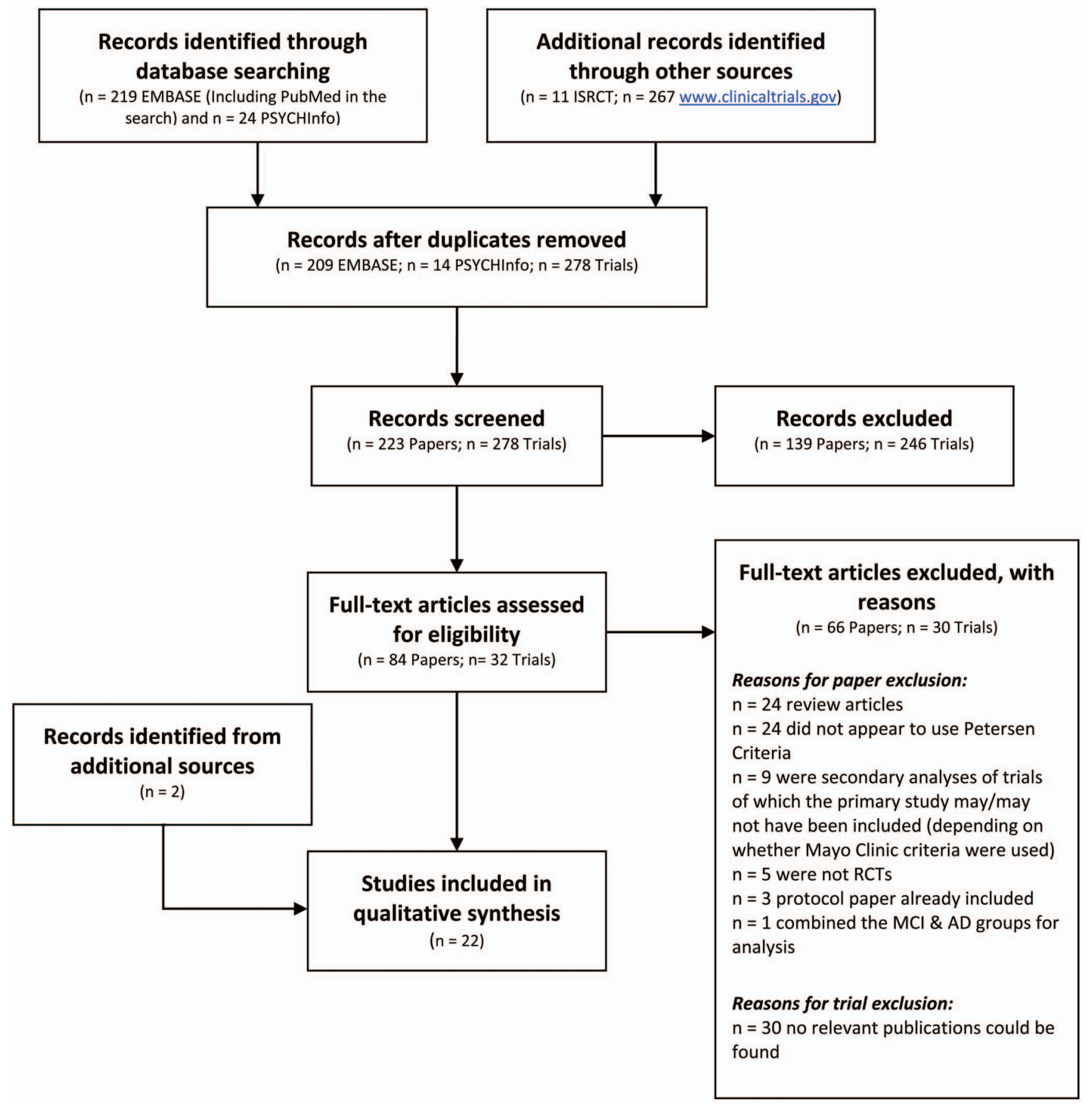

Figure 2 PRISMA (2009) flow diagram of article selection. 
galantamine, rofecoxib, fluoxetine, lithium treatment, oestrogen treatment $\left(\mathrm{E}_{2}\right)$, vitamin supplementation (E and $\mathrm{B}$ ) and supplementation with $\omega-3$ polyunsaturated fatty acids, arachidonic and docosahexaenoic acids), insulin therapy, ${ }^{18}$ physical activity ${ }^{19}{ }^{20}$ (eg, aerobic exercise), cognitive training/rehabilitation programmes $^{21-25}$ (eg, memory training, strategy learning) and combined therapies including cholinesterase inhibitor use combined with a cognitive training programme, ${ }^{26}$ and physical activity combined with vitamin B supplementation. ${ }^{27}$ Outcomes varied extensively across studies and included assessment of cognitive function (in all studies either as a primary or secondary outcome, with no neuropsychological assessment applied consistently) in addition to non-cognitive measures (eg, vascular health such as blood pressure, quality of life, depression, cerebrospinal fluid (CSF) biomarkers of Alzheimer's disease pathology and neuroimaging). Only five studies reported dementia progression rates, all of which varied: $16 \% /$ year,${ }^{9}$ 5-6\%/year, ${ }^{11} \quad 24 \%$ over 1 year, ${ }^{16} 11.9 \%$ over a 24 -week trial ${ }^{17}$ and $15 \%$ over 4 years. ${ }^{12}$ Most results were negative.

\section{Operationalising $\mathrm{MCl}$ component criterion}

Two studies ${ }^{16} 19$ did not report details of the operationalisation protocol for defining MCI.

\section{Criterion 1: memory complaint}

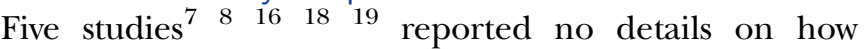
memory complaint was obtained. The memory complaint was obtained from the subject in four ${ }^{15} 212227$ studies, whereas 11 studies $^{69-11} 13141720232426$ utilised subject report and informant corroboration. One study $^{25}$ gave unclear details on who had reported the complaint. In one study, ${ }^{12}$ this criterion was operationalised using a history of gradual onset and slow progressive decline in cognitive function, but how this was reported, for example, the subject or informant, was not stated. Three studies ${ }^{10} 22{ }^{27}$ used specific scales rather than a single question to assess memory complaint. Smith et $a l^{10}$ used four items from the Cambridge Examination for Mental Disorders. ${ }^{28}$ Rapp $e t a l^{22}$ used the Memory Functioning Questionnaire, ${ }^{29}$ which is a 64-item questionnaire assessing memory problems and the use of mnemonics. Van Uffelen $e t a l^{27}$ used a positive response to a single item 'Do you have memory complaints?' or answering 'sometimes' at least twice on the cognition scale of Strawbridge. ${ }^{30}$

\section{Criterion 2: general cognitive function}

This criterion was the one most consistently measured and was typically operationalised using the Mini Mental State Examination (MMSE) ${ }^{31}$ score either alone $^{6-8} 101122$ or in combination with other measures including: a structured interview with the patient and informant, ${ }^{24}$ the Dementia Rating Scale-II ${ }^{32}$ (DRS-II), ${ }^{23}$ the Mattis DRS $^{33}$ (total score), ${ }^{14}$ the Telephone Interview for Cognitive Status ${ }^{34}$ (TICS), ${ }^{27}$ the Clinic
Dementia Rating ${ }^{35}$ (CDR) score $^{9}{ }^{26}$ or the Alzheimer's Disease Assessment Scale-Cognitive Subscale ${ }^{36}$, in addition to the Clinician Interview-Based Impression of Change $^{37}$ (CIBIC) ${ }^{17}$ One study used only the CDR score of $0.5 .^{12}$

The cut-off chosen for the MMSE varied from 23 to 26. Most studies used a cut-off value of $\geq 24,{ }^{69-11} 222627$ but $\geq 26,{ }^{7} \geq 23,{ }^{25}$ or a score adjusted for age/education, ${ }^{8} 23$ were also used. In one study, ${ }^{6}$ the protocol was modified during recruitment and the cut-off was adjusted from 24-30 to 24-28. One study ${ }^{20}$ used a 12-Item shortened MMSE with a cut-off score of $\geq 7$. Three studies ${ }^{141724}$ specified the use of the MMSE but did not report a cut-off score. Six studies did not specify operationalisation of this criterion. $^{131516181921}$

\section{Criterion 3: object memory decline}

Five studies did not specify operationalisation of this criterion. $^{78161926}$ Numerous different tests were used to assess cognition as shown in online supplementary table S2. In addition to inconsistency in test selection, there was no consistency in impairment severity (eg, 1SD, 1.5SDs or 2SDs below the mean). Further, it was not always stated whether cut-off scores for impairment were adjusted for age, education or premorbid ability. In one study, ${ }^{11}$ severity was adjusted from 1.5SDs below the mean (used in the first 6 months) to 1SD below the mean during the course of screening. Based on the nature of the objective deficit, three studies ${ }^{14} 2124$ reported the inclusion of single or multidomain aMCI. One study ${ }^{10}$ reported the use of combined amnestic and non-amnestic (single and multidomain) cases.

In terms of non-memory performance, one study ${ }^{22}$ reported that this was tested and required to be unimpaired (defined using a cut-off $>10$ th percentile). Another ${ }^{13}$ reported that performance was required to be relatively normal in non-memory domains. In one study, ${ }^{15}$ division of cases was unclear; the objective deficit in this study was defined as impairment on a total score comprising five domains (immediate and delayed memory, visuospatial/construction, language and attention) assessed using the Repeatable Battery for the Assessment of Neuropsychological Status. ${ }^{38}$

\section{Criterion 4: Activities of Daily Living (ADL)/Instrumental Activities of Daily Living (IADL)}

Seven studies did not specify operationalisation of this criterion. ${ }^{6} 81315161819$ In 11 studies, ${ }^{7} 911121721$ 23-27 minimal or non-significant functional impairment was allowed. One study required that in MCI cases that had an MMSE score between 23 and 25, cognitive impairments did not significantly interfere with daily activities or social functioning, determined by a caregiver report. ${ }^{25}$ This restriction was not required in MCI cases with a MMSE score $\geq 26$.

Functional impairment tended to be assessed by a selfreport or informant report of the difficulty with ADLs or Basic ADLs. Specific scales were used for functional 
assessment in some studies ${ }^{10} 11212627$ including: the Functional Autonomy Measurement System ${ }^{39}$ (SMAFQ), the Blessed Dementia Rating Scale (BDRS)-CERAD ${ }^{40}$ version, the Groningen Activity Restriction Scale ${ }^{41}$ and selected items from the Lawton ${ }^{42}$ and $\mathrm{Katz}^{43}$ scales or items from the Cambridge Behavioural Inventory ${ }^{44}$ (CBI). In only two studies did it appear that no evidence of any functional impairment was allowed; one, ${ }^{10}$ based on five items, related to ADLs from the CBI, and another $^{20}$ specified no decline in ADLs without their measurement being specified.

\section{Criterion 5: dementia diagnosis}

Three studies did not specify operationalisation of this criterion. $^{71419}$ Fourteen $^{6} 8-11131517182021{ }^{24-26}$ studies used the Diagnostic and Statistical Manual (DSM-III-R/ IV-TR/-IV), ${ }^{45}$ National Institute of Neurological and Communicative Disorders and Stroke and the Alzheimer's Disease and Related Disorders Association $\left(\right.$ NINCDS-ADRDA) ${ }^{47}$ criteria or National Institute of Neurological Disorders and Stroke and Association Internationale pour la Recherché et l'Enseignement en Neurosciences (NINCDS-AIREN) ${ }^{48}$ criteria. Two studies used the CDR score, ${ }^{12} 16$ and one each used a self-report of a diagnosis, ${ }^{22}$ clinical judgement ${ }^{23}$ or the TICS combined with a MMSE score $<24$. $^{27}$

\section{Additional measures}

In some studies, additional measures, generally related to the assessment of global functioning (such as the CDR sum of boxes score) or dementia severity (eg, from none, mild, moderate and severe), were made in parallel to the mapping of the five aMCI criteria. For example, two studies $^{1921}$ administered the DRS, seven ${ }^{6-12} 26$ the CDR, one $^{11}$ the BDRS, ${ }^{40}$ one $^{17}$ the CIBIC, and one ${ }^{25}$ the Global Deterioration Scale $^{49}$ (GDS). One study ${ }^{1050}$ also had informants complete both the Informant Questionnaire on Cognitive Decline in the Elderly ${ }^{51}$ (IQCODE-Short form) and EuroQol ${ }^{52}$ (EQ-5D), a measure of health status.

\section{DISCUSSION}

This review highlights the lack of consistency in MCI case ascertainment in currently completed RCTs. How MCI was diagnosed was not always reported or clear, and varying operationalisation protocols make it impossible to determine similarity across the samples recruited in the different trials. A priority for clinical trial research is to agree on a uniform set of criteria to operationalise MCI. The recruitment protocols identified in this review could provide the basis for future work to determine best practice (eg, in terms of testing classification accuracy of the different methods used), in order to inform the development of a consistent recruitment methodology for MCI clinical trials.

The review highlights the continuing challenge of operationalising the current Petersen $e t a l^{3}$ definition of aMCI. Without a standard operationalisation protocol for defining aMCI cases clinical trial recruitment will continue to be variable. Indeed, within the field of dementia, there is a lack of consistency in operationalisation protocols not only for aMCI, but its associated disorders (eg, Cognitive Impairment no Dementia ${ }^{53}$ ), dementia and its subtypes (such as Alzheimer's disease, Lewy Body dementia, frontotemporal dementia and vascular dementia), pre-MCI ${ }^{54}$ and other predementia states such as VCIND. ${ }^{55}$ For some dementias and their related conditions, it may however be difficult and unrealistic to have one set of operational criteria, precise assessment instruments or cut-off values. For example, a single set of criteria may not be possible for defining symptom fluctuations (eg, as seen in Lewy Body dementia), capturing variability in symptom profiles (eg, the different type of aphasic deficit presented in frontotemporal dementia) or reflecting differences in neuropathological profiles (eg, for vascular dementia and VCIND, the type and location of vascular damage may result in variable symptom profiles). Different diagnostic criteria for MCI affect prevalence ${ }^{56}$ and progression. ${ }^{57}$ Similarly, for dementia, different criteria have been found to affect prevalence. ${ }^{58}{ }^{59}$ Inconsistency in case classification for any health condition, whether it is within the field of dementia or any other disease category, can have an impact on research and trial recruitment and outcomes.

With regard to aMCI, consensus needs to be reached on five core issues relating to the measurement of each of the component criteria. First, whether memory complaint should be self-reported and/or informant reported and how it should be assessed (eg, single or multiple items). Second, how global cognitive function should be assessed with possible measures including the MMSE, CDR and GDS, and what the best cut-off score is (within and across cultures). Third, which neuropsychological test(s) should be used to assess memory, ${ }^{60}$ what should be the severity of cognitive impairment (1SD, $1.5 \mathrm{SD})$ and whether covariate adjustment is needed. In addition, there is the question of whether both memory and non-memory domains should be tested. The possible tests identified in this review are outlined in online supplementary table S2. Fourth, how functional performance should be assessed (the type of questions), the nature of the task (eg, instrumental ADLs, basic ADLs), reporting (eg, patient, informant or clinician) and what is the maximum level of impairment (eg, none, mild, moderate or severe difficulty or significant difficulty in some areas but not in others). Fifth, how should dementia be defined for exclusion with examples used including: the DSM or NINCDS-ADRDA criteria, the CDR sum of boxes score $\geq 1$ or via screening instruments (eg, the Telephone Screening Instrument). It should be noted that aMCI is not always operationalised as originally specified (eg, permissible significant functional impairment in some studies), and consensus needs to be reached on whether all five criteria are necessary. Further, whether modifications (if any) to criteria can be made and the 
implications of making modifications, for example, in terms of dementia predictability and effect on generalisability, needs to be established.

Decision also needs to be reached on the best treatment target. The impairment captured in aMCI is not always progressive, with a proportion of cases reverting to normal or remaining stable at follow-up, particularly when mapped in population-based studies. ${ }^{57} 61$ Indeed, symptoms of MCI are not always a consequence of Alzheimer's pathology, but rather can have multiple aetiologies such as depression or vascular disease, each with different outcomes (eg, dementia progression, improvement with treatment for the underlying health symptoms). ${ }^{62}{ }^{63}$ Better methods are needed to determine the underlying cause of disease in this patient group to accurately identify those individuals whose MCI is associated with Alzheimer's disease. One possibility could be defining aMCI as in the Alzheimer's Disease Cooperative Study trial ${ }^{9}$ (based on a subjective memory complaint, MMSE score, impaired performance on the Logical Memory II Subscale, no functional impairment and a CDR score of 0.5) as strict implementation of this methodology has been found to result in a consistent rate of dementia progression (approximately 16\%/year) across studies, including the multicentre Alzheimer's Disease Neuroimaging Initiative. ${ }^{64}$ Further research is needed to test this method of operationalisation across cohorts (clinical and population based; across countries) and calculate prevalence and longitudinal course in order to determine the generalisability of these findings. Such results could have important implications in terms of identifying a standard protocol for all future aMCI clinical trials.

A recent task force on designing trials in early (predementia) Alzheimer's disease (AD) argues for the use of aMCI criteria in combination with biomarkers to improve case selection for clinical trials. ${ }^{2}{ }^{65}$ Suggestions for possible biomarkers have included hippocampal or whole brain atrophy, CSF A $\beta 42$ levels, PiB imaging, genetic screening (APOE e4 status) or behavioural deficits, ${ }^{66-68}$ as each has been associated with dementia. Further, how dementia and $\mathrm{AD}$ are defined is currently undergoing revision, with the aim of improved stratification of patients. ${ }^{65}{ }^{69}$ Where MCI now sits in the ever changing 'lexicon' of AD (ie, given there is currently no concrete border between preclinical and clinical disease) will have implications for who is targeted for clinical trial recruitment. For example, MCI, as defined by Petersen et al criteria, may no longer be considered at-risk, but as already $\mathrm{AD}$ and encompassed in the new-term 'prodromal AD'; an early symptomatic stage where a patient shows evidence of memory impairment and positive ratings on pathophysiological and topographical markers of $\mathrm{AD} .{ }^{65}$ Clinical trial research may therefore shift some focus to asymptomatic at-risk states (eg, pre-MCI) where individuals are biomarker positive for AD but are otherwise healthy. However, like aMCI efforts are needed to standardise criteria and develop an operational protocol for any new stage of disease (eg, prodromal $\mathrm{AD}$ and pre-MCI) and to undertake validation across settings including oldest-old age groups and populations (vs clinical samples).

The review should be viewed in light of some limitations. First, we chose to focus on Petersen et al-defined aMCI, as this is one of the commonly applied definitions in clinical and research practice. However, not all trials on preclinical cognitive states have used this definition of MCI, with some studies defining intermediate cognitive states using simply a MMSE score or using criteria that have made refinements to the original aMCI criteria. ${ }^{70}$ The main change has been in the acceptable level of functional impairment: from none to allowing minor problems, particularly in complex activities such as, for example, account keeping. Different definitions of MCI have different prevalence estimates ${ }^{56}$ and also vary in their risk of dementia progression (eg, more extensive patterns of cognitive changes have been associated with greater progression of MCI to dementia).$^{57}$ Subtypes have also been defined depending on the neuropsychological profile including amnestic and nonamnestic single or multidomain MCI, and multidomain combined MCI that includes both memory and nonmemory deficits. Which, if any, of the many different criteria $^{72}$ and subtypes should be adopted in RCTs, or whether no distinction should be made between MCI and $\mathrm{AD}$ during recruitment ${ }^{2}$ requires further discussion.

\section{CONCLUSION}

Much work needs to be done on the characterisation of individuals at risk of dementia for clinical trial recruitment. Within this framework, attention is being focused on redefining the earliest stages of disease and generating new definitions of what constitutes 'prodromal/pre-dementia' and 'at-risk'. Standardisation in the definition and development of an operational protocol will result in improvements in diagnosis and clinical trial methodology.

Contributors ICMJE authorship was met by all authors. BS and TM designed the review. BS, TM, EP and MS contributed to a review of article selection, data extraction and the writing of the results section. BS, TM and MS wrote the first draft of the paper. CB and IM made substantial contribution to the intellectual content. All authors contributed to the final version and approve its submission.

Funding This research received no specific grant from any funding agency in the public, commercial or not-for-profit sectors. The authors were personally salaried by their institutions during the period of writing (though no specific salary was set aside or given for the writing of this paper).

Competing interests None.

Provenance and peer review Not commissioned; externally peer reviewed.

Data sharing statement The manuscript is a systematic review. The review protocol is available on request from the corresponding author.

\section{REFERENCES}

1. Petersen RC. Mild cognitive impairment clinical trials. Nat Rev Drug Discov 2003;2:646-53. 
2. Aisen PS, Andrieu S, Sampaio C, et al. Report of the task force on designing clinical trials in early (predementia) AD. Neurology 2011;76:280-6.

3. Petersen RC, Smith GE, Waring SC, et al. Mild cognitive impairment: clinical characterization and outcome. Arch Neurol 1999:56:303-8.

4. Petersen RC, Doody R, Kurz A, et al. Current concepts in mild cognitive impairment. Arch Neurol 2001;58:1985-92.

5. Moher D, Liberati A, Tetzlaff J, et al. Preferred reporting items for systematic reviews and meta-analyses: the PRISMA statement. PLoS Med 2009;6:e1000097.

6. Doody RS, Ferris SH, Salloway S, et al. Donepezil treatment of patients with $\mathrm{MCl}$ : a 48-week randomized, placebo-controlled trial. Neurology 2009;72:1555-61.

7. Koontz J, Baskys A. Effects of galantamine on working memory and global functioning in patients with mild cognitive impairment: a double-blind placebo-controlled study. Am J Alzheimers Dis Other Dementias 2005;20:295-302.

8. Mowla A, Mosavinasab M, Pani A. Does fluoxetine have any effect on the cognition of patients with mild cognitive impairment? A double-blind, placebo-controlled, clinical trial. J Clin Psychopharmacol 2007;27:67-70.

9. Petersen RC, Thomas RG, Grundman M, et al. Vitamin E and donepezil for the treatment of mild cognitive impairment. $N$ Engl J Med 2005;352:2379-88.

10. Smith AD, Smith SM, de Jager CA, et al. Homocysteine-lowering by $b$ vitamins slows the rate of accelerated brain atrophy in mild cognitive impairment: a randomized controlled trial. PLoS One 2010;5:1-10.

11. Thal LJ, Ferris SH, Kirby L, et al. A randomized, double-blind, study of rofecoxib in patients with mild cognitive impairment. Neuropsychopharmacology 2005;30:1204-15.

12. Winblad B, Gauthier S, Scinto L, et al. Safety and efficacy of galantamine in subjects with mild cognitive impairment. Neurology 2008;70:2024-35.

13. Chiu CC, Su KP, Cheng TC, et al. The effects of omega-3 fatty acids monotherapy in Alzheimer's disease and mild cognitive impairment: a preliminary randomized double-blind placebo-controlled study. Prog Neuropsychopharmacol Biol Psychiatry 2008;32:1538-44.

14. Chen X, Magnotta VA, Duff K, et al. Donepezil effects on cerebral blood flow in older adults with mild cognitive deficits.

J Neuropsychiatry Clin Neurosci 2006;18:178-85.

15. Kotani S, Sakaguchi E, Warashina S, et al. Dietary supplementation of arachidonic and docosahexaenoic acids improves cognitive dysfunction. Neurosci Res 2006;56:159-64.

16. Forlenza OV, Diniz BS, Radanovic M, et al. Disease-modifying properties of long-term lithium treatment for amnestic mild cognitive impairment: randomised controlled trial. Br J Psychiatry 2011;198:351-6.

17. Sherwin $\mathrm{BB}$, Chertkow $\mathrm{H}$, Schipper $\mathrm{H}$, et al. A randomized controlled trial of estrogen treatment in men with mild cognitive impairment. Neurobiol Aging 2011;32:1808-17.

18. Craft S, Baker LD, Montine TJ, et al. Intranasal insulin therapy for Alzheimer disease and amnestic mild cognitive impairment: a pilot clinical trial. Arch Neurol 2012;69:29-38.

19. Baker LD, Frank LL, Foster-Schubert K, et al. Effects of aerobic exercise on mild cognitive impairment: a controlled trial. Arch Neurol 2010;67:71-9.

20. Scherder EJ, Van Paasschen J, Deijen JB, et al. Physical activity and executive functions in the elderly with mild cognitive impairment. Aging Ment Health 2005;9:272-80.

21. Jean L, Simard M, Wiederkehr S, et al. Efficacy of a cognitive training programme for mild cognitive impairment: results of a randomised controlled study. Neuropsychol Rehabil 2010;20:377-405.

22. Rapp S, Brenes G, Marsh AP. Memory enhancement training for older adults with mild cognitive impairment: a preliminary study. Aging Mental Health 2002;6:5-11.

23. Troyer AK, Murphy KJ, Anderson ND, et al. Changing everyday memory behaviour in amnestic mild cognitive impairment: a randomised controlled trial. Neuropsychol Rehabil 2008;18:65-88.

24. Kinsella GJ, Mullaly E, Rand E, et al. Early intervention for mild cognitive impairment: a randomised controlled trial. J Neurol Neurosurg Psychiatry 2009;80:730-6.

25. Buschert VC, Friese U, Teipel SJ, et al. Effects of a newly developed cognitive intervention in amnestic mild cognitive impairment and mild Alzheimer's disease: a pilot study. J Alzheimers Dis 2011;25:679-94

26. Rozzini L, Costardi D, Chilovi V, et al. Efficacy of cognitive rehabilitation in patients with mild cognitive impairment treated with cholinesterase inhibitors. Int J Geriatric Psychiatry 2007;22:356-60.
27. Van Uffelen JGZ, Hopman-Rock M, Chin A, et al Protocol for Project FACT: a randomised controlled trial on the effect of a walking program and vitamin $\mathrm{B}$ supplementation on the rate of cognitive decline and psychosocial wellbeing in older adults with mild cognitive impairment [ISRCTN19227688]. BMC Geriatrics 2005;5. doi:10.1186/1471-2318-1185-1118

28. Roth M, Tym E, Mountjoy $\mathrm{CQ}$, et al. CAMDEX: the cambridge examination for mental disorders of the elderly. Cambridge: Cambridge University Press, 1988.

29. Gilewski MJ, Zelinski EM. Memory Functioning Questionnaire (MFQ). Psychopharmacol Bull 1988;24:665-70.

30. Strawbridge WJ, Shema SJ, Balfour JL, et al. Antecedents of frailty over three decades in an older cohort. J Gerontol Series B, Psychol Sci Soc Sci 1998;53:S9-16.

31. Folstein MF, Folstein SE, McHugh PR. 'Mini-mental state'. A practical method for grading the cognitive state of patients for the clinician. J Psychiatr Res 1975;12:189-98.

32. Jurica PJ, Leitten CL, Mattis S. Dementia rating scale-2: professional manual. Lutz: Psychological Assessment Resources, 2001.

33. Mattis S. Dementia rating scale: orofessional manual. Odessa, FL: Psychological Assessment Resources, 1988.

34. Brandt J, Spencer M, Folstein M. The telephone interview for cognitive status. Neuropsychiatry Neuropsychol Behav Neurol 1988;1:111-17.

35. Hughes CP, Berg L, Danziger WL, et al. A new clinical scale for the staging of dementia. Br J Psychiatry 1982;140:566-72.

36. Rosen WG, Mohs RC, Davis KL. A new rating scale for Alzheimer's disease. Am J Psychiatry 1984;141:1356-64.

37. Schneider LS, Olin JT, Doody RS, et al. Validity and reliability of the Alzheimer's disease cooperative study-clinical global impression of change. The Alzheimer's Disease Cooperative Study. Alzheimer Dis Assoc Disord 1997;11:S22-32.

38. Randolph C, Tierney MC, Mohr E, et al. The Repeatable Battery for the Assessment of Neuropsychological Status (RBANS): preliminary clinical validity. J Clin Exp Neuropsychol 1998;20:310-19.

39. Hebert R, Carrier R, Bilodeau A. The Functional Autonomy Measurement System (SMAF): description and validation of an instrument for the measurement of handicaps. Age Ageing 1988;17:293-302.

40. Morris JC, Mohs RC, Rogers $\mathrm{H}$, et al. Consortium to establish a registry for Alzheimer's disease (CERAD) clinical and neuropsychological assessment of Alzheimer's disease. Psychopharmacol Bull 1988;24:641-52.

41. Kempen GI, Miedema I, Ormel J, et al. The assessment of disability with the Groningen activity restriction scale. Conceptual framework and psychometric properties. Soc Sci Med 1996;43:1601-10.

42. Lawton MP, Brody EM. Assessment of older people: self-maintaining and instrumental activities of daily living. Gerontologist 1969:9:179-86.

43. Katz S, Downs TD, Cash HR, et al. Progress in development of the index of ADL. Gerontologist 1970;10:20-30.

44. Wedderburn $\mathrm{C}$, Wear $\mathrm{H}$, Brown J, et al. The utility of the Cambridge Behavioural Inventory in neurodegenerative disease. J Neurol Neurosurg Psychiatry 2008;79:500-3.

45. American Psychiatric Association. Diagnostic and statistical manual of mental disorders (Third Edition, revised) (DSM-III-R). Washington, DC: APA, 1987

46. American Psychiatric Association. Diagnostic and statistical manual of mental disorders (Fourth Edition) (Text Revision: DMS-IV-TR) Arlington, VA: American Psychiatric Publishing Inc, 2000.

47. McKhann G, Drachman D, Folstein M, et al. Clinical diagnosis of Alzheimer's disease: report of the NINCDS-ADRDA Work Group under the auspices of Department of Health and Human Services Task Force on Alzheimer's Disease. Neurology 1984;34:939-44.

48. Roman GC, Tatemichi TK, Erkinjuntti T, et al. Vascular dementia: diagnostic criteria for research studies. Report of the NINDS-AIREN International Workshop. Neurology 1993;43:250-60.

49. Reisberg B, Ferris SH, de Leon MJ, et al. The Global Deterioration Scale for assessment of primary degenerative dementia. Am J Psychiatry 1982;139:1136-9.

50. de Jager CA, Oulhaj A, Jacoby $R$, et al. Cognitive and clinical outcomes of homocysteine-lowering B-vitamin treatment in mild cognitive impairment: a randomized controlled trial. Int J Geriatric Psychiatry 2012;26:592-600.

51. Jorm AF. The Informant Questionnaire on cognitive decline in the elderly (IQCODE): a review. Int Psychogeriatr 2004;16:275-93.

52. EuroQol Group. EuroQol-A new facility for the measurement of health-related quality of life. Health Policy 1990;16:199-208. 
53. Ebly EM, Hogan DB, Parhad IM. Cognitive impairment in the nondemented elderly. Results from the Canadian Study of Health and Aging. Arch Neurol 1995;52:612-19.

54. Duara R, Loewenstein DA, Greig MT, et al. Pre-MCl and $\mathrm{MCl}$ : neuropsychological, clinical, and imaging features and progression rates. Am J Geriatric Psychiatry 2011;19:951-60.

55. Stephan BC, Matthews FE, Khaw KT, et al. Beyond mild cognitive impairment: vascular cognitive impairment, no dementia (VCIND). Alzheimer's Res Ther 2009;1:4.

56. Stephan BC, Matthews FE, McKeith IG, et al. Early cognitive change in the general population: how do different definitions work? J Am Geriatr Soc 2007:55:1534-40.

57. Matthews FE, Stephan BC, McKeith IG, et al. Two-year progression from mild cognitive impairment to dementia: to what extent do different definitions agree? J Am Geriatr Soc 2008;56:1424-33.

58. Erkinjuntti T, Ostbye T, Steenhuis R, et al. The effect of different diagnostic criteria on the prevalence of dementia. $N$ Engl J Med 1997;337:1667-74.

59. Wancata J, Borjesson-Hanson A, Ostling S, et al. Diagnostic criteria influence dementia prevalence. Am J Geriatric Psychiatry 2007;15:1034-45.

60. Lonie JA, Tierney KM, Ebmeier KP. Screening for mild cognitive impairment: a systematic review. Int J Geriatr Psychiatry 2009;24:902-15.

61. Mitchell AJ, Shiri-Feshki M. Rate of progression of mild cognitive impairment to dementia-meta-analysis of 41 robust inception cohort studies. Acta Psychiatrica Scandinavica 2009;119:252-65.

62. DeCarli C. Mild cognitive impairment: prevalence, prognosis, aetiology, and treatment. Lancet Neurol 2003;2:15-21.

63. Panza F, Capurso C, D'Introno A, et al. Heterogeneity of mild cognitive impairment and other predementia syndromes in progression to dementia. Neurobiol Aging 2007;28:1631-2.
64. Petersen RC, Aisen PS, Beckett LA, et al. Alzheimer's Disease Neuroimaging Initiative (ADNI): clinical characterization. Neurology 2010;74:201-9.

65. Dubois $\mathrm{B}$, Feldman $\mathrm{HH}$, Jacova $\mathrm{C}$, et al. Revising the definition of Alzheimer's disease: a new lexicon. Lancet Neurol 2010;9:1118-27.

66. Whitehair DC, Sherzai A, Emond J, et al. Influence of apolipoprotein e (epsilon)4 on rates of cognitive and functional decline in mild cognitive impairment. Alzheimer's Dementia 2010;6:412-19.

67. Jack CR Jr, Knopman DS, Jagust WJ, et al. Hypothetical model of dynamic biomarkers of the Alzheimer's pathological cascade. Lancet Neurol 2010;9:119-28.

68. Gauthier S, Reisberg B, Zaudig M, et al. Mild cognitive impairment. Lancet 2006;367:1262-70.

69. Sperling RA, Aisen PS, Beckett LA, et al. Toward defining the preclinical stages of Alzheimer's disease: recommendations from the National Institute on Aging-Alzheimer's Association workgroups on diagnostic guidelines for Alzheimer's disease. Alzheimers Dement 2011;7:280-92.

70. Winblad B, Palmer K, Kivipelto M, et al. Mild cognitive impairmentbeyond controversies, towards a consensus: report of the International Working Group on Mild Cognitive Impairment. J Intern Med 2004;256:240-6.

71. Albert MS, DeKosky ST, Dickson D, et al. The diagnosis of mild cognitive impairment due to Alzheimer's disease: recommendations from the National Institute on Aging-Alzheimer's Association workgroups on diagnostic guidelines for Alzheimer's disease. Alzheimers Dement 2011;7:270-9.

72. Matthews FE, Stephan BC, Bond J, et al. Operationalization of mild cognitive impairment: a graphical approach. PLoS Med 2007;4:1615-19. 